\title{
A Novel $\mathbf{X}^{-}(\mathrm{X}=\mathrm{I}, \mathrm{Br})$-Triggered Ring-opening Coupling Reaction of Cyclopropenes with Organic Halides.
}

Shengming Ma*, Junliang Zhang, Yangjun Cai, and Lianghua Lu

\section{Supporting Information}

Typical procedure for ring-opening coupling reaction of cyclopropenes with organic halides.

(1) (E)-2-Allyl-2-(2'-iodovinyl)malonic acid dimethyl ester (3aa).

A mixture of cyclopropene 1a $(78 \mathrm{mg}, 0.5 \mathrm{mmol})$, allyl iodide $\mathbf{2 a}(168 \mathrm{mg}, 1.0$ mmol), $\mathrm{NaI}(7.5 \mathrm{mg}, 10 \mathrm{~mol} \%), \mathrm{Na}_{2} \mathrm{CO}_{3}(27 \mathrm{mg}, 50 \mathrm{~mol} \%)$, and acetone $(2 \mathrm{~mL})$ was stirred under reflux for $2.5 \mathrm{~h}$. After evaporation, the residue was purified by column chromatography (eluent: petroleum ether $\left./ \mathrm{Et}_{2} \mathrm{O}=10 / 1\right)$ on silica gel to give $\mathbf{3 a a}(111 \mathrm{mg}$, $69 \%)$ as a liquid. ${ }^{1} \mathrm{H} \mathrm{NMR}\left(\mathrm{CDCl}_{3}, 300 \mathrm{MHz}\right): \delta 6.93(\mathrm{~d}, J=15.3 \mathrm{~Hz}, 1 \mathrm{H}), 6.42(\mathrm{~d}, J=$ $15.3 \mathrm{~Hz}, 1 \mathrm{H}), 5.72-5.56(\mathrm{~m}, 1 \mathrm{H}), 5.18-5.04(\mathrm{~m}, 2 \mathrm{H}), 3.74(\mathrm{~s}, 6 \mathrm{H}), 2.78(\mathrm{dt}, J=7.5$ and $1.2 \mathrm{~Hz}, 2 \mathrm{H}) ;{ }^{13} \mathrm{C} \mathrm{NMR}\left(\mathrm{CDCl}_{3}, 75.4 \mathrm{MHz}\right): \delta 169.0,140.6,131.6,119.6,79.9,62.5$, 52.9, 39.2; MS (70 eV): m/z (\%): 325(15.34) [ $\left.M^{+}+1\right], 197$ (100); IR (neat): 1736, 1642, $1600,1241,1209,948,927 \mathrm{~cm}^{-1}$; Anal. calcd for $\mathrm{C}_{10} \mathrm{H}_{13} \mathrm{IO}_{4}$ : C 37.06, H 4.04. Found: $\mathrm{C}$ 37.32, H 4.18 .

(2) (E)-2-Benzyl-2-(2'-iodovinyl)malonic acid dimethyl ester (3ab).

The reaction of $1 \mathbf{a}(78 \mathrm{mg}, 0.5 \mathrm{mmol})$, benzyl iodide $2 \mathbf{b}(218 \mathrm{mg}, 1.0 \mathrm{mmol}), \mathrm{NaI}$ (7.5 mg, $10 \mathrm{~mol} \%$ ), and $\mathrm{Na}_{2} \mathrm{CO}_{3}(27 \mathrm{mg}, 50 \mathrm{~mol} \%)$ in $2 \mathrm{~mL}$ of acetone under reflux afforded 3ab $(151 \mathrm{mg}, 81 \%)$ as a liquid. ${ }^{1} \mathrm{H}$ NMR $\left(\mathrm{CDCl}_{3}, 300 \mathrm{MHz}\right): \delta 7.30-7.20(\mathrm{~m}, 3$ H), 7.10-7.02 (m, $2 \mathrm{H}), 6.84(\mathrm{~d}, J=15.3 \mathrm{~Hz}, 1 \mathrm{H}), 6.38(\mathrm{~d}, J=15.3 \mathrm{~Hz}, 1 \mathrm{H}), 3.73(\mathrm{~s}, 6$ $\mathrm{H}), 3.36(\mathrm{~s}, 2 \mathrm{H}) ;{ }^{13} \mathrm{C} \mathrm{NMR}\left(\mathrm{CDCl}_{3}, 75.4 \mathrm{MHz}\right): \delta 169.1,141.3,134.7,129.9,128.2$, 127.2, 79.3, 63.8, 52.8, 42.0; MS (70 eV): m/z (\%): 375 (4.07) [M+1], 91 (100); IR (neat): $1736,1600,1496,1445,1435,1271,1198,949 \mathrm{~cm}^{-1}$; Anal. calcd for $\mathrm{C}_{14} \mathrm{H}_{15} \mathrm{IO}_{4}$ : C 44.94, H 4.04. Found: C 45.14, H 4.20.

(3) (E)-2-(2'-Iodovinyl)-2-(2''-oxo-2''-phenylethyl)malonic acid dimethyl ester (3ac).

The reaction of 1a (78 mg, $0.5 \mathrm{mmol}), \alpha$-iodoacetophenone $2 \mathbf{c}(246 \mathrm{mg}, 1.0 \mathrm{mmol})$, $\mathrm{NaI}(7.5 \mathrm{mg}, 10 \mathrm{~mol} \%)$, and $\mathrm{Na}_{2} \mathrm{CO}_{3}(27 \mathrm{mg}, 50 \mathrm{~mol} \%)$ in $2 \mathrm{~mL}$ of acetone under reflux afforded 3ac $(161 \mathrm{mg}, 80 \%)$ as colorless needles. m.p. $132-133^{\circ} \mathrm{C}\left(\mathrm{Et}_{2} \mathrm{O}\right.$-hexanes); ${ }^{1} \mathrm{H}$ NMR ( $\left.\mathrm{CDCl}_{3}, 300 \mathrm{MHz}\right): \delta$ 8.02-7.94 (m, $\left.2 \mathrm{H}\right), 7.64-7.56(\mathrm{~m}, 1 \mathrm{H}), 7.54-7.44(\mathrm{~m}, 2 \mathrm{H})$, $7.50(\mathrm{~d}, J=15.3 \mathrm{~Hz}, 1 \mathrm{H}), 6.35(\mathrm{~d}, J=15.3 \mathrm{~Hz}, 1 \mathrm{H}), 3.92(\mathrm{~s}, 2 \mathrm{H}), 3.81(\mathrm{~s}, 6 \mathrm{H}) ;{ }^{13} \mathrm{C}$ NMR $\left(\mathrm{CDCl}_{3}, 75.4 \mathrm{MHz}\right): \delta$ 195.4, 168.9, 141.0, 135.8, 133.7, 128.7, 128.1, 79.0, 59.8, 53.4, 44.7; MS (70 eV): m/z (\%): $401(0.03)\left[M^{+}-1\right], 105$ (100); IR (KBr): 1763, 1732, 
1686, 1599, 1280, 1249, 959, $933 \mathrm{~cm}^{-1}$; Anal. calcd for $\mathrm{C}_{15} \mathrm{H}_{15} \mathrm{IO}_{5}: \mathrm{C} 44.80, \mathrm{H} 3.76$. Found: C 44.80, H 3.74.

(4) (E)-2-(2'-iodovinyl)-2-(ethoxycarbonylmethyl)malonic acid dimethyl ester (3ad).

The reaction of $\mathbf{1 a}(78 \mathrm{mg}, 0.5 \mathrm{mmol}), \alpha$-iodoacetate $\mathbf{2 d}(214 \mathrm{mg}, 1.0 \mathrm{mmol}), \mathrm{NaI}$ (7.5 mg, $10 \mathrm{~mol} \%$ ), and $\mathrm{Na}_{2} \mathrm{CO}_{3}(27 \mathrm{mg}, 50 \mathrm{~mol} \%)$ in $2 \mathrm{~mL}$ of acetone under reflux afforded 3ad (120 mg, 65\%) as an oil. ${ }^{1} \mathrm{H} \mathrm{NMR}\left(\mathrm{CDCl}_{3}, 300 \mathrm{MHz}\right): \delta 7.09(\mathrm{~d}, J=15.0$ Hz, $1 \mathrm{H}), 6.44$ (d, J = 15.0 Hz, $1 \mathrm{H}), 4.12$ (q, J= 7.5 Hz, $2 \mathrm{H}), 3.78$ (s, $6 \mathrm{H}), 3.14$ (s, $2 \mathrm{H})$, $1.23(\mathrm{t}, J=7.5 \mathrm{~Hz}, 3 \mathrm{H}) ;{ }^{13} \mathrm{C} \mathrm{NMR}\left(\mathrm{CDCl}_{3}, 75.4 \mathrm{MHz}\right): \delta 169.4,168.3,140.2,79.7,61.1$, 59.9, 53.3, 39.3, 14.0; MS (70 eV): m/z (\%): 371 (20.00) $\left[M^{+}+1\right], 243$ (100); IR (neat): $1735,1600,1436,1280,1199,1027,950 \mathrm{~cm}^{-1}$; Anal. calcd for $\mathrm{C}_{11} \mathrm{H}_{15} \mathrm{IO}_{6}: \mathrm{C} 35.69, \mathrm{H}$ 4.08. found: C 35.92, H 4.18.

(5) (E)- 2-(2'-Iodovinyl)-2-oct-2''-ynyl-malonic acid dimethyl ester (3ae).

The reaction of $1 \mathrm{a}(78 \mathrm{mg}, 0.5 \mathrm{mmol}), 1$-iodooct-2-yne $2 \mathbf{e}(236 \mathrm{mg}, 1.0 \mathrm{mmol}), \mathrm{NaI}$ (7.5 mg, $10 \mathrm{~mol} \%$ ), and $\mathrm{Na}_{2} \mathrm{CO}_{3}(27 \mathrm{mg}, 50 \mathrm{~mol} \%)$ in $2 \mathrm{~mL}$ of acetone under reflux afforded 3ae $(161 \mathrm{mg}, 82 \%)$ as a liquid. ${ }^{1} \mathrm{H} \mathrm{NMR}\left(\mathrm{CDCl}_{3}, 300 \mathrm{MHz}\right): \delta 6.97(\mathrm{~d}, J=15.0$ $\mathrm{Hz}, 1 \mathrm{H}), 6.48$ (d, $J=15.0 \mathrm{~Hz}, 1 \mathrm{H}), 3.79$ (s, $6 \mathrm{H}), 2.89$ (t, $J=2.4 \mathrm{~Hz}, 2 \mathrm{H}), 2.10$ (tt, $J=$ 7.5 and $2.4 \mathrm{~Hz}, 2 \mathrm{H}), 1.50-1.40(\mathrm{~m}, 2 \mathrm{H}), 1.40-1.24(\mathrm{~m}, 4 \mathrm{H}), 0.91(\mathrm{t}, J=7.2 \mathrm{~Hz}, 3 \mathrm{H})$; ${ }^{13} \mathrm{C} \mathrm{NMR}\left(\mathrm{CDCl}_{3}, 75.4 \mathrm{MHz}\right): \delta 168.5,140.0,84.4,80.1,73.6,62.4,53.1,30.8,28.4$, 25.0, 22.2, 18.5, 14.0; MS (70 eV): m/z (\%): 393 (4.54) $\left[M^{+}+1\right], 145$ (100); IR (neat): 1740, 1600, 1436, 1203, $944 \mathrm{~cm}^{-1}$; Anal. calcd for $\mathrm{C}_{15} \mathrm{H}_{21} \mathrm{IO}_{4}$ : C 45.93, H 5.40. Found: C 45.88, H 5.45.

(6) (E)- 2-(2'-Iodovinyl)-2-(2'-methyl-allyl)malonic acid dimethyl ester (3af).

The reaction of $\mathbf{1 a}(78 \mathrm{mg}, 0.5 \mathrm{mmol}), 3$-iodo-2-methylpropene $\mathbf{2 f}$ (182 $\mathrm{mg}, 1.0$ mmol), $\mathrm{NaI}$ (7.5 mg, $10 \mathrm{~mol} \%$ ), and $\mathrm{Na}_{2} \mathrm{CO}_{3}(27 \mathrm{mg}, 50 \mathrm{~mol} \%)$ in $2 \mathrm{~mL}$ of acetone under reflux afforded 3af $(120 \mathrm{mg}, 71 \%)$ as a liquid. ${ }^{1} \mathrm{H} \mathrm{NMR}\left(\mathrm{CDCl}_{3}, 300 \mathrm{MHz}\right): \delta 7.03(\mathrm{~d}, J$ $=15.3 \mathrm{~Hz}, 1 \mathrm{H}), 6.39(\mathrm{~d}, J=15.3 \mathrm{~Hz}, 1 \mathrm{H}), 4.83(\mathrm{t}, J=1.2 \mathrm{~Hz}, 1 \mathrm{H}), 4.65(\mathrm{~s}, 1 \mathrm{H}), 3.72(\mathrm{~s}$, $6 \mathrm{H}), 2.81(\mathrm{~s}, 2 \mathrm{H}), 1.64(\mathrm{~s}, 3 \mathrm{H}) ;{ }^{13} \mathrm{C} \mathrm{NMR}\left(\mathrm{CDCl}_{3}, 75.4 \mathrm{MHz}\right): \delta 169.3,141.0,139.7$, 115.4, 79.6, 62.0, 52.9, 43.0, 23.6; MS (70 eV): m/z (\%): 339 (10.08) [ $\left.M^{+}+1\right], 151(100)$; IR (neat): 1737, 1646, 1598, 1436, 1270, 1236, 1201, 1179, 951, $902 \mathrm{~cm}^{-1}$; HRMS calcd for $\mathrm{C}_{10} \mathrm{H}_{12} \mathrm{IO}_{3}\left[M^{+}-\mathrm{CH}_{3} \mathrm{O}\right]$ : 306.9831 . Found: 306.9868 .

(7) $(E, \quad E)$-2-(2'-Iodovinyl)-5-(3'-methoxycarbonyl-prop-2''-enyl)malonic acid dimethyl ester (3ag).

The reaction of $\mathbf{1 a}(78 \mathrm{mg}, 0.5 \mathrm{mmol})$, methyl 4-iodocrotonate $\mathbf{2 g}$ (226 $\mathrm{mg}, 1.0$ mmol), $\mathrm{NaI}$ (7.5 mg, $10 \mathrm{~mol} \%$ ), and $\mathrm{Na}_{2} \mathrm{CO}_{3}(27 \mathrm{mg}, 50 \mathrm{~mol} \%)$ in $2 \mathrm{~mL}$ of acetone under reflux afforded 3ag $(151 \mathrm{mg}, 79 \%)$ as a liquid. ${ }^{1} \mathrm{H} \mathrm{NMR}\left(\mathrm{CDCl}_{3}, 300 \mathrm{MHz}\right): \delta 6.88(\mathrm{~d}, J$ $=15.3 \mathrm{~Hz}, 1 \mathrm{H}), 6.70(\mathrm{dt}, J=15.9$ and $7.5 \mathrm{~Hz}, 1 \mathrm{H}), 6.42(\mathrm{~d}, J=15.3 \mathrm{~Hz}, 1 \mathrm{H}), 5.83(\mathrm{dt}, J$ $=15.9$ and $1.5 \mathrm{~Hz}, 1 \mathrm{H}), 3.71(\mathrm{~s}, 6 \mathrm{H}), 3.67(\mathrm{~s}, 3 \mathrm{H}), 2.88(\mathrm{dd}, J=7.5$ and $1.5 \mathrm{~Hz}, 2 \mathrm{H})$; ${ }^{13} \mathrm{C} \mathrm{NMR}\left(\mathrm{CDCl}_{3}, 75.4 \mathrm{MHz}\right): \delta 168.4,165.9,141.6,139.8,124.9,80.6,61.9,53.1,51.5$, 37.2; MS (70 eV): m/z (\%): 382 (0.74) [ $\left.M^{+}\right], 255$ (100); IR (neat): 1735, 1660, 1600, 1436, 1341, 1275, 1175, $983 \mathrm{~cm}^{-1}$; HRMS calcd for $\mathrm{C}_{12} \mathrm{H}_{15} \mathrm{IO}_{6}$ : 381.9913. Found: 381.9938 . 
(8) (E,E)-2-(2'-Iodovinyl)-2-(3''-phenyl-2''-propenyl)malonic acid dimethyl ester (3ah).

The reaction of $\mathbf{1 a}(78 \mathrm{mg}, 0.5 \mathrm{mmol})$, trans-cinnamy iodide $\mathbf{2 h}(244 \mathrm{mg}, 1.0 \mathrm{mmol})$, $\mathrm{NaI}(7.5 \mathrm{mg}, 10 \mathrm{~mol} \%)$, and $\mathrm{Na}_{2} \mathrm{CO}_{3}(27 \mathrm{mg}, 50 \mathrm{~mol} \%)$ in $2 \mathrm{~mL}$ of acetone under reflux afforded 3ah (183 mg, 92\%) as colorless needles. m.p. 80-81 ${ }^{\circ} \mathrm{C}\left(\mathrm{Et}_{2} \mathrm{O}\right.$-hexanes); ${ }^{1} \mathrm{H}$ NMR $\left(\mathrm{CDCl}_{3}, 300 \mathrm{MHz}\right): \delta$ 7.40-7.20 (m, $\left.5 \mathrm{H}\right), 7.00(\mathrm{~d}, J=15.0 \mathrm{~Hz}, 1 \mathrm{H}), 6.49(\mathrm{~d}, J=15.0 \mathrm{~Hz}$, $1 \mathrm{H}), 6.46(\mathrm{~d}, J=15.6 \mathrm{~Hz}, 1 \mathrm{H}), 6.04(\mathrm{dt}, J=15.6$ and $7.5 \mathrm{~Hz}, 1 \mathrm{H}), 3.75(\mathrm{~s}, 6 \mathrm{H}), 2.95(\mathrm{~d}$, $J=7.5 \mathrm{~Hz}, 2 \mathrm{H}) ;{ }^{13} \mathrm{C} \mathrm{NMR}\left(\mathrm{CDCl}_{3}, 75.4 \mathrm{MHz}\right): \delta 168.9,140.6,136.6,134.4,128.4$, 127.4, 126.1, 122.8, 80.0, 62.7, 52.9, 38.5; MS (70 eV): m/z (\%): $400(0.01)\left[M^{+}\right], 117$ (100); IR (KBr): 1736, 1599, 1578, 1496, 1435, 1266, 1237, 1200, 1180, 968, $950 \mathrm{~cm}^{-1}$; Anal. calcd for $\mathrm{C}_{16} \mathrm{H}_{17} \mathrm{IO}_{4}$ : C 48.02, H 4.28. Found: C 48.24, $\mathrm{H} 4.09$.

(9) (E)-2-Benzyl-2-(2'-bromovinyl)malonic acid dimethyl ester (3ai).

The reaction of $1 \mathrm{a}(78 \mathrm{mg}, 0.5 \mathrm{mmol})$, benzyl bromide $2 \mathbf{i}(257 \mathrm{mg}, 1.5 \mathrm{mmol}), \mathrm{LiBr}$ (4.4 mg, $10 \mathrm{~mol} \%$ ), and $\mathrm{Li}_{2} \mathrm{CO}_{3}(19 \mathrm{mg}, 50 \mathrm{~mol} \%$ ) in $2 \mathrm{~mL}$ of acetone under reflux afforded 3ai (110 mg, 67\%) as colorless needles. m.p. $63-64^{\circ} \mathrm{C}$ (Et $t_{2} \mathrm{O}$-hexanes); ${ }^{1} \mathrm{H}$ NMR $\left(\mathrm{CDCl}_{3}, 300 \mathrm{MHz}\right): \delta$ 7.36-7.20 (m, $\left.3 \mathrm{H}\right), 7.12-7.06(\mathrm{~m}, 2 \mathrm{H}), 6.50(\mathrm{~d}, J=14.1 \mathrm{~Hz}, 1 \mathrm{H})$, $6.30(\mathrm{~d}, J=14.1 \mathrm{~Hz}, 1 \mathrm{H}), 3.75$ (s, $6 \mathrm{H}), 3.38(\mathrm{~s}, 2 \mathrm{H}) ;{ }^{13} \mathrm{C} \mathrm{NMR}\left(\mathrm{CDCl}_{3}, 75.4 \mathrm{MHz}\right): \delta$ 169.3, 134.8, 133.7, 130.0, 128.3, 127.3, 109.0, 61.9, 53.0, 42.2; MS (70 eV): m/z (\%): 247 (7.69) $\left[M^{+}+1-\mathrm{Br}\right], 91$ (100); IR (KBr): 1738, 1614, 1585, 1496, 1455, 1435, 1273, 1207, 1179, 1083, $936 \mathrm{~cm}^{-1}$; Anal. calcd for $\mathrm{C}_{14} \mathrm{H}_{15} \mathrm{BrO}_{4}$ : C 51.40, H 4.62. Found: $\mathrm{C}$ $51.42, \mathrm{H} 4.46$.

(10) $(E, E)-2-(2$ '-Bromovinyl)-2-(3"'-phenylprop-2"-enyl)malonic acid dimethyl ester (3aj).

The reaction of 1a $(78 \mathrm{mg}, 0.5 \mathrm{mmol}$ ), trans-cinnamyl bromide $\mathbf{2 \mathbf { j }}$ (197 $\mathrm{mg}, 1.0$ $\mathrm{mmol}), \mathrm{LiBr}(8.7 \mathrm{mg}, 20 \mathrm{~mol} \%)$, and $\mathrm{Li}_{2} \mathrm{CO}_{3}(19 \mathrm{mg}, 50 \mathrm{~mol} \%)$ in $2 \mathrm{~mL}$ of acetone under reflux afforded 3aj (113 mg, 64\%) as colorless needles. m.p. 52-53 ${ }^{\circ} \mathrm{C}\left(\mathrm{Et}_{2} \mathrm{O}-\right.$ hexanes); ${ }^{1} \mathrm{H}$ NMR ( $\left.\mathrm{CDCl}_{3}, 300 \mathrm{MHz}\right): \delta 7.38-7.20(\mathrm{~m}, 5 \mathrm{H}), 6.65(\mathrm{~d}, J=14.1 \mathrm{~Hz}, 1 \mathrm{H}), 6.47(\mathrm{dt}, J=$ 15.6 and $1.2 \mathrm{~Hz}, 1 \mathrm{H}), 6.40(\mathrm{~d}, J=14.1 \mathrm{~Hz}, 1 \mathrm{H}), 6.04(\mathrm{dt}, J=15.6$ and $7.2 \mathrm{~Hz}, 1 \mathrm{H})$, 3.77 (s, $6 \mathrm{H}), 2.96(\mathrm{dd}, J=7.2$ and $1.2 \mathrm{~Hz}, 2 \mathrm{H}) ;{ }^{13} \mathrm{C} \mathrm{NMR}\left(\mathrm{CDCl}_{3}, 75.4 \mathrm{MHz}\right): \delta 169.2$, 136.7, 134.5, 133.2, 128.5, 127.6, 126.2, 122.8, 109.6, 60.9, 53.0, 38.8; MS (70 eV): m/z (\%): $354(0.18)\left[M^{+}\left({ }^{81} \mathrm{Br}\right)\right], 352(0.20)\left[M^{+}\left({ }^{79} \mathrm{Br}\right)\right], 117$ (100); IR (neat): 1737, 1616, 1600, 1496, 1436, 1268, 1239, 1209, 1181, 969, $942 \mathrm{~cm}^{-1}$; Anal. calcd for $\mathrm{C}_{16} \mathrm{H}_{17} \mathrm{BrO}_{4}: \mathrm{C}$ 54.41, H 4.85. Found: C 54.51, H 4.93 .

(11) (E)-2-Allyl-2-(1'-iodomethylenepentyl)malonic acid dimethyl ester.

The reaction of $\mathbf{1 b}(106 \mathrm{mg}, 0.5 \mathrm{mmol})$, allyl iodide $\mathbf{2 a}(168 \mathrm{mg}, 1.0 \mathrm{mmol}), \mathrm{NaI}$ (7.5 $\mathrm{mg}, 10 \mathrm{~mol} \%$ ), and $\mathrm{Na}_{2} \mathrm{CO}_{3}(27 \mathrm{mg}, 50 \mathrm{~mol} \%)$ in $2 \mathrm{~mL}$ of acetone under reflux afforded 3ba (171 mg, 90\%) as a liquid. ${ }^{1} \mathrm{H}$ NMR $\left(\mathrm{CDCl}_{3}, 300 \mathrm{MHz}\right): \delta 6.60(\mathrm{~s}, 1 \mathrm{H}), 5.84-5.64$ $(\mathrm{m}, 1 \mathrm{H}), 5.14-5.02(\mathrm{~m}, 2 \mathrm{H}), 3.71(\mathrm{~s}, 6 \mathrm{H}), 2.79(\mathrm{dt}, J=6.9$ and $1.2 \mathrm{~Hz}, 2 \mathrm{H}), 2.21(\mathrm{t}, J=$ $7.5 \mathrm{~Hz}, 2 \mathrm{H}) ; 1.48-1.20(\mathrm{~m}, 4 \mathrm{H}), 0.90(\mathrm{t}, J=7.8 \mathrm{~Hz}, 3 \mathrm{H}) ;{ }^{13} \mathrm{C} \mathrm{NMR}\left(\mathrm{CDCl}_{3}, 75.4 \mathrm{MHz}\right)$ : $\delta$ 169.5, 144.9, 132.8, 118.9, 83.5, 65.2, 52.5, 39.1, 36.4, 29.7, 23.0, 13.7; ESI-MS: 403.1 
$\left[M+\mathrm{Na}^{+}\right]$; IR (neat): 1737, 1640, 1596, 1232, $1218 \mathrm{~cm}^{-1}$; HRMS calcd for $\mathrm{C}_{14} \mathrm{H}_{21} \mathrm{O}_{4} \mathrm{Na}$ $\left[M+\mathrm{Na}^{+}\right]:$403.0382. Found: 403.0368.

(12) (E)-2-Benzyl-2-(1'-iodomethylenepentyl)malonic acid dimethyl ester (3ba).

The reaction of $\mathbf{1 b}(106 \mathrm{mg}, 0.5 \mathrm{mmol})$, benzyl iodide $\mathbf{2 b}(218 \mathrm{mg}, 1.0 \mathrm{mmol}), \mathrm{NaI}$ (7.5 mg, $10 \mathrm{~mol} \%$ ), and $\mathrm{Na}_{2} \mathrm{CO}_{3}(27 \mathrm{mg}, 50 \mathrm{~mol} \%)$ in $2 \mathrm{~mL}$ of acetone under reflux afforded $3 \mathbf{b b}(213 \mathrm{mg}, 99 \%)$ as an oil. ${ }^{1} \mathrm{H} \mathrm{NMR}\left(\mathrm{CDCl}_{3}, 300 \mathrm{MHz}\right): \delta 7.30-7.20(\mathrm{~m}, 3 \mathrm{H})$, 7.10-7.02 (m, $2 \mathrm{H}), 6.55$ (s, $1 \mathrm{H}), 3.68(\mathrm{~s}, 6 \mathrm{H}), 3.39$ (s, $2 \mathrm{H}), 2.24(\mathrm{t}, J=7.2 \mathrm{~Hz}, 2 \mathrm{H})$, $1.52-1.28(\mathrm{~m}, 4 \mathrm{H}), 0.94(\mathrm{t}, J=7.2 \mathrm{~Hz}, 3 \mathrm{H}) ;{ }^{13} \mathrm{C} \mathrm{NMR}\left(\mathrm{CDCl}_{3}, 75.4 \mathrm{MHz}\right): \delta 169.3$, $143.5,135.5,130.2,127.9,127.0,85.2,66.1,52.4,40.8,36.6,29.7,23.0,13.7$; MS (70 $\mathrm{eV}): \mathrm{m} / \mathrm{z}(\%): 430(0.53)\left[M^{+}+1\right], 77$ (100); IR (neat): 1740, 1604, 1496, 1456, 1434, 1262, 1170, $1085 \mathrm{~cm}^{-1}$; HRMS calcd for $\mathrm{C}_{18} \mathrm{H}_{23} \mathrm{O}_{4}\left[M^{+}-\mathrm{I}\right]: 303.15964$. Found: 303.15912 . (13) (E,E)-2-(1'-Bromomethylenepentyl)-2-(3"-phenyl-prop-2'-enyl)malonic acid dimethyl ester (3bj).

The reaction of $\mathbf{1 b}(106 \mathrm{mg}, 0.5 \mathrm{mmol})$, trans-cinnamyl bromide $\mathbf{2 \mathbf { j }}$ (197 $\mathrm{mg}, 1.0$ mmol), $\mathrm{LiBr}(8.7 \mathrm{mg}, 20 \mathrm{~mol} \%)$, and $\mathrm{Li}_{2} \mathrm{CO}_{3}(19 \mathrm{mg}, 50 \mathrm{~mol} \%)$ in $2 \mathrm{~mL}$ of acetone under reflux afforded $3 \mathbf{b j}(175 \mathrm{mg}, 86 \%)$ as an oil. ${ }^{1} \mathrm{H} \mathrm{NMR}\left(\mathrm{CDCl}_{3}, 300 \mathrm{MHz}\right): \delta 7.36-7.18(\mathrm{~m}$, $5 \mathrm{H}), 6.54(\mathrm{~s}, 1 \mathrm{H}), 6.43(\mathrm{~d}, J=15.9 \mathrm{~Hz}, 1 \mathrm{H}), 6.16(\mathrm{dt}, J=15.9,7.2 \mathrm{~Hz}, 1 \mathrm{H}), 3.72(\mathrm{~s}, 6$ H), $2.95(\mathrm{~d}, J=7.2 \mathrm{~Hz}, 2 \mathrm{H}), 2.25(\mathrm{t}, J=8.1 \mathrm{~Hz}, 2 \mathrm{H}), 1.54-1.41(\mathrm{~m}, 2 \mathrm{H}), 1.41-1.24(\mathrm{~m}$, $2 \mathrm{H}), 0.92(\mathrm{t}, J=7.5 \mathrm{~Hz}, 3 \mathrm{H}) ;{ }^{13} \mathrm{C} \mathrm{NMR}\left(\mathrm{CDCl}_{3}, 75.4 \mathrm{MHz}\right): \delta 169.5,140.4,137.0$, 133.9, 128.4, 127.4, 126.2, 124.2, 109.7, 65.0, 52.6, 38.2, 32.5, 29.6, 23.1, 13.7; MS (70 $\mathrm{eV}): \mathrm{m} / \mathrm{z}(\%): 411(1.08)\left[M^{+}\left({ }^{81} \mathrm{Br}\right)+1\right], 409 \quad\left[M^{+}\left({ }^{79} \mathrm{Br}\right)+1\right], 117$ (100); IR (neat): 1737, $1600,1578,1496,1435,1262,1235,969 \mathrm{~cm}^{-1}$; Anal. calcd for $\mathrm{C}_{20} \mathrm{H}_{25} \mathrm{BrO}_{4}: \mathrm{C} 58.69, \mathrm{H}$ 6.16. Found: C 58.66, H 6.18.

(14) (E)-2-Allyl-2-(benzenesulfonyl)-3-(iodomethylene)heptanoic acid ethyl ester.

The reaction of 1c (154 mg, $0.5 \mathrm{mmol})$, allyl iodide $2 \mathbf{a}$ (336 mg, $2.0 \mathrm{mmol}), \mathrm{NaI}$ (15 $\mathrm{mg}, 20 \mathrm{~mol} \%)$, and $\mathrm{Na}_{2} \mathrm{CO}_{3}(27 \mathrm{mg}, 50 \mathrm{~mol} \%)$ in $2 \mathrm{~mL}$ of acetone under reflux afforded 3ca $(131 \mathrm{mg}, 64 \%)$ as an oil. ${ }^{1} \mathrm{H}$ NMR $\left(\mathrm{CDCl}_{3}, 300 \mathrm{MHz}\right): \delta 7.85-7.80(\mathrm{~m}, 2 \mathrm{H}), 7.66-$ $7.58(\mathrm{~m}, 1 \mathrm{H}), 7.55-7.44(\mathrm{~m}, 2 \mathrm{H}), 6.57(\mathrm{~s}, 1 \mathrm{H}), 6.00-5.84(\mathrm{~m}, 1 \mathrm{H})$, 5.24-5.12 (m, $2 \mathrm{H})$, 4.28-4.12 (m, $2 \mathrm{H}), 3.28(\mathrm{dd}, J=14.7$ and $6.0 \mathrm{~Hz}, 1 \mathrm{H}), 3.00(\mathrm{dd}, J=14.7$ and $7.8 \mathrm{~Hz}, 1$ H), 2.14-1.96 (m, 2 H), 1.34-1.16 (m, $4 \mathrm{H}), 1.26(\mathrm{t}, J=7.2 \mathrm{~Hz}, 3 \mathrm{H}), 0.82(\mathrm{t}, J=7.2 \mathrm{~Hz}, 3$ $\mathrm{H}) ;{ }^{13} \mathrm{C} \mathrm{NMR}\left(\mathrm{CDCl}_{3}, 75.4 \mathrm{MHz}\right): \delta 166.6,142.0,136.5,133.9,132.0,131.4,128.2$, 119.7, 89.5, 81.7, 62.3, 37.0, 36.5, 29.3, 22.8, 13.7, 13.6; MS (70 eV): m/z (\%): $477(0.35)$ $\left[M^{+}+1\right], 335$ (100); IR (neat): 1736, 1639, 1584, 1466, 1488, 1310, 1212, 1150, 1080, $923 \mathrm{~cm}^{-1}$; Anal. calcd for $\mathrm{C}_{19} \mathrm{H}_{25} \mathrm{IO}_{4} \mathrm{~S}$ : C 47.90, H 5.29. Found: C 47.78, H 5.32.

\section{Synthetic transformations of 3aa.}

(1) (E)-2-Allyl-2-styrylmalonic acid dimethyl ester (4aa).

A mixture of 3aa $(81 \mathrm{mg}, 0.25 \mathrm{mmol})$, phenylboronic acid (61 $\mathrm{mg}, 0.5 \mathrm{mmol})$, $\mathrm{Pd}\left(\mathrm{PPh}_{3}\right)_{4}(14 \mathrm{mg}, 5 \mathrm{~mol} \%), \mathrm{Na}_{2} \mathrm{CO}_{3}(2 \mathrm{M}, 0.3 \mathrm{~mL}), \mathrm{CH}_{3} \mathrm{OH}(0.1 \mathrm{~mL})$ and toluene $(2 \mathrm{~mL})$ was refluxed under Ar for $5 \mathrm{~h}$. After extraction with ether, the combined extracts were washed with $\mathrm{H}_{2} \mathrm{O}$ and saturated $\mathrm{NaCl}$ successively. After drying over $\mathrm{MgSO}_{4}$, filtration, and evaporation, the residue was purified by column chromatography on silica gel to 
afford 4aa (49 mg, $72 \%$ ) as an oil. ${ }^{1} \mathrm{H}$ NMR $\left(\mathrm{CDCl}_{3}, 300 \mathrm{MHz}\right): \delta 7.46-7.39(\mathrm{~m}, 2 \mathrm{H})$, 7.38-7.24 (m, $3 \mathrm{H}), 6.70(\mathrm{~d}, J=16.5 \mathrm{~Hz}, 1 \mathrm{H}), 6.52(\mathrm{~d}, J=16.5 \mathrm{~Hz}, 1 \mathrm{H}), 5.80-5.66(\mathrm{~m}, 1$ $\mathrm{H}), 5.18-5.06(\mathrm{~m}, 2 \mathrm{H}), 3.77(\mathrm{~s}, 6 \mathrm{H}), 2.94(\mathrm{dt}, J=7.2$ and $1.2 \mathrm{~Hz}, 2 \mathrm{H}) ;{ }^{13} \mathrm{C} \mathrm{NMR}$ $\left(\mathrm{CDCl}_{3}, 75.4 \mathrm{MHz}\right): \delta 170.5,136.3,132.2,131.8,128.5,128.0,126.5,125.7,119.1,59.6$, 52.7, 40.0; MS (70 eV): m/z (\%): 274 (1.19) [ $\left.M^{+}\right], 173$ (100); IR (neat): 1735, 1642, 1601, 1579, 1497, 1436, 1232, 1208, $967 \mathrm{~cm}^{-1}$; HRMS calcd for $\mathrm{C}_{16} \mathrm{H}_{18} \mathrm{O}_{4}$ : 274.1205. Found: 274.1515.

(2) (E)-2-Allyl-2-(4'-phenylbut-1'-en-3'-ynyl)malonic acid dimethyl ester (5aa).

A mixture of 3aa $(81 \mathrm{mg}, 0.25 \mathrm{mmol})$, phenylacetylene $(51 \mathrm{mg}, 0.5 \mathrm{mmol})$, $\mathrm{PdCl}_{2}\left(\mathrm{PPh}_{3}\right)_{2}(8.8 \mathrm{mg}, 5 \mathrm{~mol} \%), \mathrm{CuI}(4.8 \mathrm{mg}, 10 \mathrm{~mol} \%), \mathrm{K}_{2} \mathrm{CO}_{3}(69 \mathrm{mg}, 0.5 \mathrm{mmol})$, and $\mathrm{CH}_{3} \mathrm{CN}(2 \mathrm{~mL})$ was stirred at $\mathrm{rt}$ under Ar for $6 \mathrm{~h}$. Evaporation and purification by column chromatography on silica gel afforded 5aa $(68 \mathrm{mg}, 91 \%)$ as colorless needles. m.p. 73$74^{\circ} \mathrm{C}$ (Et ${ }_{2} \mathrm{O}$-hexanes). ${ }^{1} \mathrm{H}$ NMR $\left(\mathrm{CDCl}_{3}, 300 \mathrm{MHz}\right): \delta 7.50-7.40(\mathrm{~m}, 2 \mathrm{H}), 7.36-7.28(\mathrm{~m}, 3$ $\mathrm{H}), 6.62(\mathrm{~d}, J=16.5 \mathrm{~Hz}, 1 \mathrm{H}), 5.87(\mathrm{~d}, J=16.5 \mathrm{~Hz}, 1 \mathrm{H}), 5.76-5.60$ (m, $1 \mathrm{H}), 5.18-5.08$ $(\mathrm{m}, 2 \mathrm{H}), 3.75(\mathrm{~s}, 6 \mathrm{H}), 2.86(\mathrm{~d}, J=7.2 \mathrm{~Hz}, 2 \mathrm{H}) ;{ }^{13} \mathrm{C} \mathrm{NMR}\left(\mathrm{CDCl}_{3}, 75.4 \mathrm{MHz}\right): \delta 169.7$, $138.4,131.7,131.4,128.33,128.25,122.9,119.4,112.6,91.4,86.8,59.9,52.8,39.6$; MS (70 eV): m/z (\%): 298 (11.13) [ $\left.M^{+}\right], 179$ (100); IR (KBr): 1750, 1729, 1641, 1232, 1210, $963,923 \mathrm{~cm}^{-1}$; Anal. calcd for $\mathrm{C}_{18} \mathrm{H}_{18} \mathrm{O}_{4}$ : C 72.47, H 6.08. Found: C 72.57, H 6.31.

(3) (E)-2-Allyl-2-(hex-1'-enyl)malonic acid dimethyl ester (6aa).

To a solution of anhydrous $\mathrm{ZnBr}_{2}(270 \mathrm{mg}, 1.2 \mathrm{mmol})$ in $2 \mathrm{~mL}$ of THF was added dropwise $0.63 \mathrm{~mL}$ of $\mathrm{BuLi}(1.6 \mathrm{M}$ in hexanes, $1.0 \mathrm{mmol})$ at $0^{\circ} \mathrm{C}$. After stirring for $5 \mathrm{~min}$, $\mathrm{Pd}\left(\mathrm{PPh}_{3}\right)_{4}(29 \mathrm{mg}, 5 \mathrm{~mol} \%)$ and 3aa $(162 \mathrm{mg}, 0.5 \mathrm{mmol})$ were added and the mixture was stirred for another $1 \mathrm{~h} . \mathrm{H}_{2} \mathrm{O}(5 \mathrm{~mL})$ was added and the reaction mixture was extracted by ether. After drying over $\mathrm{MgSO}_{4}$, filtration, and evaporation, the residue was purified by column chromatography on silica gel to afford 6aa (100 $\mathrm{mg}, 79 \%)$ as a liquid. ${ }^{1} \mathrm{H} \mathrm{NMR}$ $\left(\mathrm{CDCl}_{3}, 300 \mathrm{MHz}\right): \delta 5.88(\mathrm{dt}, J=15.9$ and $1.2 \mathrm{~Hz}, 1 \mathrm{H}), 5.72-5.50(\mathrm{~m}, 2 \mathrm{H}), 5.10-5.00$ $(\mathrm{m}, 2 \mathrm{H}), 3.71(\mathrm{~s}, 6 \mathrm{H}), 2.76(\mathrm{dt}, J=8.0$ and $1.2 \mathrm{~Hz}, 2 \mathrm{H}), 2.12-2.02(\mathrm{~m}, 2 \mathrm{H}), 1.38-1.22$ $(\mathrm{m}, 4 \mathrm{H}), 0.87(\mathrm{t}, J=6.9 \mathrm{~Hz}, 3 \mathrm{H}) ;{ }^{13} \mathrm{C} \mathrm{NMR}\left(\mathrm{CDCl}_{3}, 75.4 \mathrm{MHz}\right): \delta 170.8,133.3,132.5$, 125.8, 118.6, 59.3, 52.4, 39.9, 32.3, 31.0, 22.0, 13.8; MS (70 eV): m/z (\%): 255 (5.71) $\left[M^{+}+1\right], 254$ (2.99) $\left[M^{+}\right], 213$ (100); IR (neat): 1737, 1642, 1233, 1205, $970 \mathrm{~cm}^{-1}$; HRMS calcd for $\mathrm{C}_{11} \mathrm{H}_{17} \mathrm{O}_{4}\left[M^{+}-\mathrm{C}_{3} \mathrm{H}_{5}\right]: 213.1127$. Found: 213.1128 .

(4) $(E, E)-2$-Allyl-2-(4'-methoxycarbonylbuta-1',3'-dienyl)malonic acid dimethyl ester (7aa).

A mixture of 3aa (162 mg, $0.5 \mathrm{mmol})$, acrylic acid methyl ester (86 mg, $1.0 \mathrm{mmol})$, $\mathrm{Pd}\left(\mathrm{PPh}_{3}\right)_{4}(29 \mathrm{mg}, 5 \mathrm{~mol} \%), \mathrm{K}_{2} \mathrm{CO}_{3}(138 \mathrm{mg}, 1.0 \mathrm{mmol})$ and $\mathrm{CH}_{3} \mathrm{CN}(5 \mathrm{~mL})$ was stirred at $70^{\circ} \mathrm{C}$ under $\mathrm{Ar}$ for $24 \mathrm{~h}$. After evaporation, the residue was purified by column chromatography on silica gel to afford 7aa $(113 \mathrm{mg}, 79 \%)$ as colorless needles. m.p. 41$42^{\circ} \mathrm{C}$ (Et $2 \mathrm{O}$-hexanes). ${ }^{1} \mathrm{H} \mathrm{NMR}\left(\mathrm{CDCl}_{3}, 300 \mathrm{MHz}\right): \delta 7.25(\mathrm{dd}, J=15.3$ and $10.8 \mathrm{~Hz}, 1$ $\mathrm{H}), 6.46(\mathrm{~d}, J=15.9 \mathrm{~Hz}, 1 \mathrm{H}) ; 6.23(\mathrm{dd}, J=15.9$ and $10.8 \mathrm{~Hz}, 1 \mathrm{H}), 5.87(\mathrm{~d}, J=15.3 \mathrm{~Hz}$, $1 \mathrm{H}), 5.66-5.50(\mathrm{~m}, 1 \mathrm{H}), 5.10-5.00(\mathrm{~m}, 2 \mathrm{H}), 3.704(\mathrm{~s}, 6 \mathrm{H}), 3.697$ (s, $3 \mathrm{H}), 2.80$ (d, $J=$ $7.2 \mathrm{~Hz}, 2 \mathrm{H}) ;{ }^{13} \mathrm{C} \mathrm{NMR}\left(\mathrm{CDCl}_{3}, 75.4 \mathrm{MHz}\right): \delta 169.6,166.9,143.5,137.6,131.6,129.7$, 
122.1, 119.4, 59.8, 52.8, 51.5, 39.7; MS (70 eV): m/z (\%): 282 (1.16) [ $\left.M^{+}\right], 59$ (100); IR (neat): 1737, 1729, 1643,1616, 1436, 1232, 1210, 1144, $1000 \mathrm{~cm}^{-1}$; Anal. calcd for $\mathrm{C}_{14} \mathrm{H}_{18} \mathrm{O}_{6}:$ C 59.57, H 6.43. Found: C 59.32, H 6.59. 